\title{
Distribution amplitudes of pseudoscalar mesons
}

\section{QCDSF/UKQCD Collaboration: V. M. Braun ${ }^{a}$, M. Göckeler ${ }^{a}$, R. Horsley ${ }^{b}$, H. Perlt ${ }^{c}$,} D. Pleiter ${ }^{d}$, P. E. L. Rakow ${ }^{e}$, G. Schierholz ${ }^{d f}$, A. Schiller ${ }^{c}$, W. Schroers ${ }^{* d}$, H. Stüben ${ }^{g}$, and J. M. Zanotti ${ }^{b}$

${ }^{a}$ Institut für Theoretische Physik, Universität Regensburg, 93040 Regensburg, Germany

${ }^{b}$ School of Physics, University of Edinburgh, Edinburgh EH9 3JZ, UK

${ }^{c}$ Institut für Theoretische Physik, Universität Leipzig, 04109 Leipzig, Germany

${ }^{d}$ John von Neumann-Institut für Computing NIC / DESY, 15738 Zeuthen, Germany

${ }^{e}$ Theoretical Physics Division, Department of Mathematical Sciences, University of Liverpool,

Liverpool L69 3BX, UK

${ }^{f}$ Deutsches Elektronen-Synchrotron DESY, 22603 Hamburg, Germany

${ }^{g}$ Konrad-Zuse-Zentrum für Informationstechnik Berlin, 14195 Berlin, Germany

We present results for the first two moments of the distribution amplitudes of pseudoscalar mesons. Using two flavors of non-perturbatively improved clover fermions and non-perturbative renormalization of the matrix elements we perform both chiral and continuum extrapolations and compare with recent results from models and experiments.

PACS: $12.88 . \mathrm{GC}, 14.40 . \mathrm{Aq}$

XXIV International Symposium on Lattice Field Theory

July 23-28 2006

Tucson Arizona, US

${ }^{*}$ Speaker. 


\section{Introduction}

Hadronic wave functions are of crucial importance when describing exclusive and semi-exclusive reactions [1]. For detailed references and applications consult [2]. Distribution amplitudes (DAs) are related to the hadron's Bethe-Salpeter wave function, $\phi\left(x, k_{\perp}\right)$, by an integral over transverse momenta. For the leading twist meson-DAs we have

$$
\phi\left(x, \mu^{2}\right)=Z_{2}\left(\mu^{2}\right) \int_{\left|k_{\perp}\right|<\mu} d^{2} k \phi\left(x, k_{\perp}\right),
$$

where $x$ is the quark longitudinal momentum fraction, $Z_{2}$ the renormalization factor (in the lightcone gauge) for the quark-field operators in the wave-function, and $\mu$ denotes the renormalization scale. In this presentation we quote all numbers with a scale $\mu^{2}=4 \mathrm{GeV}^{2}$ in the $\overline{\mathrm{MS}}$-scheme.

It is convenient to rescale $\xi=2 x-1$. In the following we use $\phi(\xi)$ to describe any pseudoscalar meson, $\phi_{\pi}(\xi)$ to refer to the pion, and $\phi_{K}(\xi)$ to denote the kaon. Furthermore, it is common to expand DAs into their Gegenbauer moments and quote the expansion coefficients, $a_{i}$, at a given renormalization scale as a parameterization of DAs,

$$
\phi\left(\xi, \mu^{2}\right)=\frac{3}{4}\left(1-\xi^{2}\right)\left(1+\sum_{n=1}^{\infty} a_{n}\left(\mu^{2}\right) C_{n}^{3 / 2}(\xi)\right) .
$$

The zeroth moment is normalized to unity, $\int_{-1}^{1} d \xi \phi\left(\xi, \mu^{2}\right)=1$, at any energy scale $\mu^{2}$. From renormalization group arguments we find that

$$
\phi\left(\xi, \mu^{2} \rightarrow \infty\right)=\phi_{\text {as }}(\xi)=\frac{3}{4}\left(1-\xi^{2}\right) .
$$

Taking the $u$ - and $d$-quarks to be degenerate, $G$-parity implies that the pion DA is an even function of $\xi$ and hence all odd moments vanish, i.e., $a_{2 n+1}^{\pi}=0$.

Recently, we have computed the first moments of meson distribution amplitudes [2] on the lattice. Independently, a calculation of the first moment of the kaon distribution amplitude has appeared which uses a different discretization scheme and different working points [3]. In this contribution we present our calculation of the first non-vanishing moment of the pion DA, $a_{2}^{\pi}$, and the first two moments of the kaon DA, $a_{1}^{K}$ and $a_{2}^{K}$. We compare our results to previous estimates from sum rules and experiment and discuss the implications of our lattice computation.

\section{Lattice calculation}

On the lattice, one has to perform the light-cone operator product expansion to find a relation between local operators and moments of DAs w.r.t. $\xi$. To be specific, one has

$$
\begin{aligned}
\left\langle\xi^{n}\right\rangle\left(\mu^{2}\right) & =\int_{-1}^{1} d \xi \xi^{n} \phi\left(\xi, \mu^{2}\right), \\
\left\langle\Omega\left|\mathscr{O}_{\left\{v_{0} \ldots v_{n}\right\}}(0)\right| \mathrm{PS}\right\rangle & =\mathrm{i} f_{\mathrm{PS}} p_{\left\{v_{0}\right.} \ldots p_{\left.v_{n}\right\}}\left\langle\xi \xi^{n}\right\rangle \\
\mathscr{O}_{v_{0} \ldots v_{n}}(0) & =\mathrm{i}^{n}-q(0) \gamma_{v_{0}} \gamma_{5} \stackrel{\leftrightarrow}{D}_{v_{1}} \ldots \stackrel{\leftrightarrow}{D}_{v_{n}} u(0)
\end{aligned}
$$

where "PS" refers to either the pion or the kaon, and $q$ can be either a $d$ - or an $s$-quark. $\stackrel{\leftrightarrow}{D}$ is the covariant derivative and $\{\ldots\}$ denotes the symmetrization of indices and the subtraction of traces. 
The matrix element, Eq. (2.1), can be obtained from an appropriate ratio of two-point functions, consult [2] for details. For the first moment of pseudoscalar mesons containing non-degenerate mass quarks we use the following two operators

$$
\mathscr{O}_{41}^{a}=\mathscr{O}_{\{41\}}, \quad \mathscr{O}_{44}^{b}=\mathscr{O}_{\{44\}}-\frac{1}{3}\left(\mathscr{O}_{\{11\}}+\mathscr{O}_{\{22\}}+\mathscr{O}_{\{33\}}\right) .
$$

For $\mathscr{O}_{41}^{a}$ we have used the external momentum $\vec{p}=(2 \pi / L, 0,0)$ - with $L$ being the spatial lattice size - and the corresponding rotated momenta, for $\mathscr{O}_{44}^{b}$ we have taken $\vec{p}=\overrightarrow{0}$. For the second moment we have only employed $\mathscr{O}_{412}=\mathscr{O}_{\{412\}}$ with external momentum $\vec{p}=(2 \pi / L, 2 \pi / L, 0)$.

We have generated our gauge field configurations using the Wilson gauge action and two flavors of dynamical, non-perturbatively improved clover fermions. For four different values of $\beta=5.20,5.25,5.29$, and 5.40 and up to four different $\kappa$ values per $\beta$ we have produced $\mathscr{O}(2000-$ $8000)$ trajectories. Lattice spacings and spatial volumes vary between $0.075-0.123 \mathrm{fm}$ and $(1.5-$ $2.2 \mathrm{fm})^{3}$, respectively. The scale has been set using a Sommer parameter of $r_{0}=0.467 \mathrm{fm}$. For further details see [4]. Correlation functions are calculated every $10 \mathrm{HMC}$ trajectories using four different locations of the fermion source. By applying binning we obtain an effective distance of 20 trajectories. We observe that the bin size has little effect on the error, indicating that residual autocorrelations are small.

The matching between the lattice results and the $\overline{\mathrm{MS}}$-scheme has been done non-perturbatively. The renormalization procedure has been detailed in Refs. $[5,6]$ and will be discussed further in a forthcoming publication.

\section{Numerical results}

\subsection{Mass-degenerate quarks}

For mass-degenerate quarks, i.e., the pion, we compute $\left\langle\xi^{2}\right\rangle_{\pi}\left(\mu^{2}=4 \mathrm{GeV}^{2}\right)$, and from this $a_{2}^{\pi}\left(4 \mathrm{GeV}^{2}\right)$. To obtain the result at the physical pion mass we first perform a linear chiral extrapolation of $\left\langle\xi^{2}\right\rangle$ to the physical pion mass at each fixed value of $\beta$. Figure 1 shows $\left\langle\xi^{2}\right\rangle$ obtained from $\mathscr{O}_{412}$ as a function of the pion mass at a fixed value of $\beta=5.29$ with $\kappa_{\text {sea }}=\kappa_{\text {val. }}$. The dependence on the pion mass turns out to be very weak. Using chiral perturbation theory in the continuum it has been shown [7] that for small pion masses the leading logarithmic contribution can be absorbed in the pseudoscalar decay constant, $f_{\pi}$. Hence, for small values of the pion mass we expect the dependence on $m_{\pi}$ to be rather flat. However, a precise matching of lattice results and chiral perturbation theory similar to what has been done in [8] for the nucleon axial coupling, $g_{A}$, is still to be performed.

Figure 2 shows the continuum extrapolation of the results from all values of $\beta$ at the physical pion mass for $\mathscr{O}_{412}$. Scaling violations seem to be small, introducing an uncertainty of roughly $6 \%$. The continuum result reads

$$
\left\langle\xi^{2}\right\rangle_{\pi}\left(\mu^{2}=4 \mathrm{GeV}^{2}\right)=0.269(39), \quad a_{2}^{\pi}\left(4 \mathrm{GeV}^{2}\right)=0.201(114) .
$$

It is in agreement with the older quenched result $\left\langle\xi^{2}\right\rangle_{\pi}\left(4 \mathrm{GeV}^{2}\right)=0.286(49)_{-0.013}^{+0.030}$ from [9]. It is larger than the asymptotic value, $\left\langle\xi^{2}\right\rangle_{\pi}\left(\mu^{2} \rightarrow \infty\right)=0.2$, indicating that the commonly adopted asymptotic ansatz at this energy scale may not be justified quantitatively. 


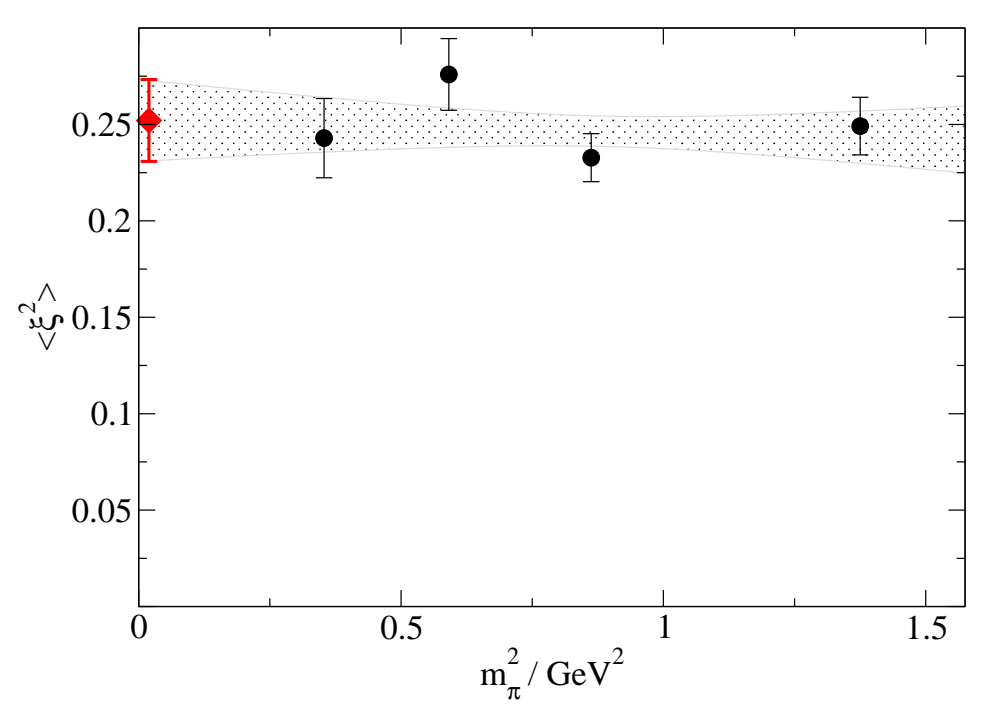

Figure 1: Pion mass dependence of the second moment of the pion distribution amplitude, $\left\langle\xi^{2}\right\rangle_{\pi}$, at $\beta=$ 5.29 , for all four values of $\kappa_{\text {sea }}=\kappa_{\text {val }}$ from $\mathscr{O}_{412}$.

The coefficient $a_{2}^{\pi}$ has been under experimental scrutiny recently. The decay of neutral pions into two photons contains information on the pion wavefunction. However, as has been reported in [10] the leading twist expression is insufficient to determine $a_{2}^{\pi}$ unambiguously. QCD sum rules, e.g. $[11,12,13]$ have found that $a_{2}$ is indeed positive and the more recent calculations in this approach $[12,13]$ are compatible with our result also in magnitude. However, the contribution of $a_{4}^{\pi}$ is expected to be non-negligible in modeling the pion DA. This makes an independent lattice analysis of this quantity particularly interesting.

\subsection{Mass non-degenerate quarks}

In case of two quarks with distinct masses, the odd moments will no longer vanish. Since we are primarily interested in the light pseudoscalar mesons we tune the parameters and extrapolations in such a manner that the light quark (corresponding to $\kappa_{\text {sea }}$ ) has the correct mass to reproduce a pion if the quarks were degenerate and the heavy quark (corresponding to $\kappa_{\text {val }}$ ) then reproduces the kaon mass. Since we require a large set of different valence masses, we have restricted ourselves to the four data sets at $\beta=5.29$ only. These have $\kappa_{\text {sea }}=0.1340,0.1350,0.1355$, and 0.1359 . The systematic error due to the continuum extrapolation is estimated from the extrapolation in Fig. 2 to be about $6 \%$.

A sample plot for the second moment of the kaon distribution amplitude can be seen in Fig. 3 for the working point $\beta=5.29, \kappa_{\text {sea }}=0.1359$. Several different values have been chosen for the heavy quark mass. The vertical line indicates the physical kaon mass and the data point at this location has been taken from a linear extrapolation.

The data from all four samples of gauge fields at $\beta=5.29$ has been combined and linearly 


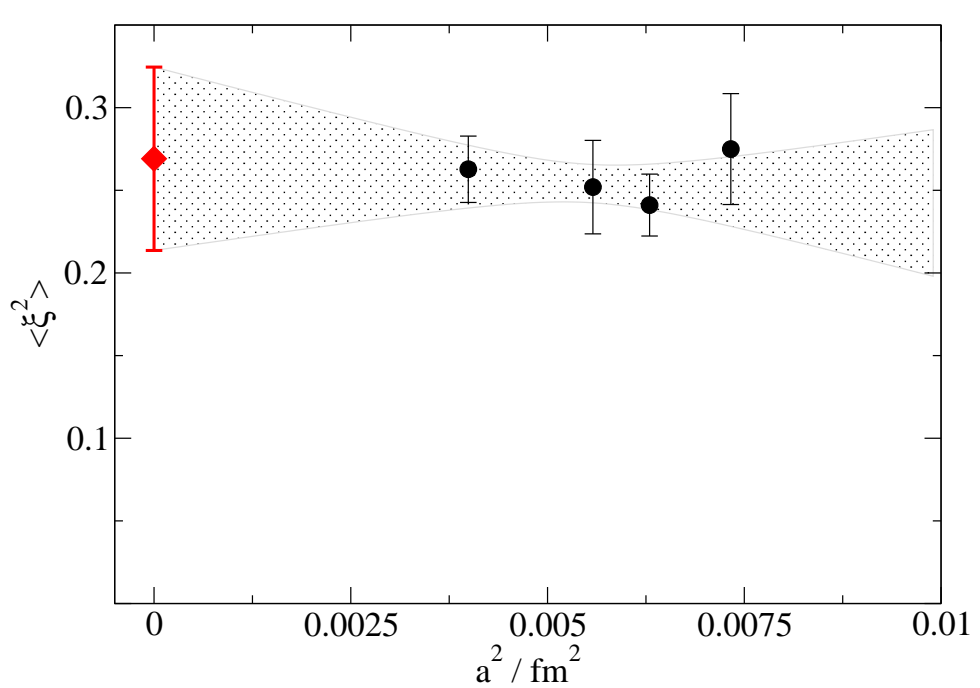

Figure 2: Continuum extrapolation of $\left\langle\xi^{2}\right\rangle_{\pi}$ for all values of $\beta$ for $\mathscr{O}_{412}$.

extrapolated to the physical values of $m_{K}$ and $m_{\pi}$ in a global fit, see [2] for details. The resulting value is

$$
\left\langle\xi^{2}\right\rangle_{K}\left(4 \mathrm{GeV}^{2}\right)=0.260(6)(16), \quad a_{2}^{K}\left(4 \mathrm{GeV}^{2}\right)=0.175(18)(47) .
$$

The first error is of statistical origin while the second one corresponds to a scaling error of $6 \%$. The result (3.2) corresponds to a ratio of $\left\langle\xi^{2}\right\rangle_{K} /\left\langle\xi^{2}\right\rangle_{\pi} \simeq 1$. In the literature, there has been the debate between [11] predicting a ratio of $a_{2}^{K} / a_{2}^{\pi} \sim 0.59 \pm 0.04$ and [12, 14] predicting a ratio of about $\sim 1$. Our finding clearly favors the latter results.

Of particular interest is the first moment of the kaon DA, $\langle\xi\rangle_{K}$, and, hence, also $a_{1}^{K}$. Figure 4 shows the interpolation of $\langle\xi\rangle$ obtained from $\mathscr{O}_{44}^{b}$ as a function of the mass difference $m_{K}^{2}-m_{\pi}^{2}$ for one sample of gauge fields. Note that this is one of the few cases where an interpolation suffices. We now have a result at a specific pion mass determined by $\kappa_{\text {sea }}=\kappa_{\text {light }}$. After repeating this procedure on our other data-sets with $\beta=5.29$, we extrapolated the results linearly in the sea quark mass to the physical pion mass. Combining this result with the value obtained from a similar analysis using $\mathscr{O}_{41}^{a}$, we finally find

$$
\langle\xi\rangle_{K}\left(4 \mathrm{GeV}^{2}\right)=0.0275(5)(17), \quad a_{1}^{K}\left(4 \mathrm{GeV}^{2}\right)=0.0453(9)(29) .
$$

Again, the first error is statistical and the second systematic. The magnitude of this quantity has been debated in the literature quite recently, see the discussion in [12]. The value we find is more accurate than the sum rule estimate $a_{1}^{K}=0.05(25)$, but fully compatible both in sign and in magnitude. The independent investigation in [3] finds a value of $a_{1}^{K}\left(4 \mathrm{GeV}^{2}\right)=0.053(5)$ which is also compatible with our finding. 


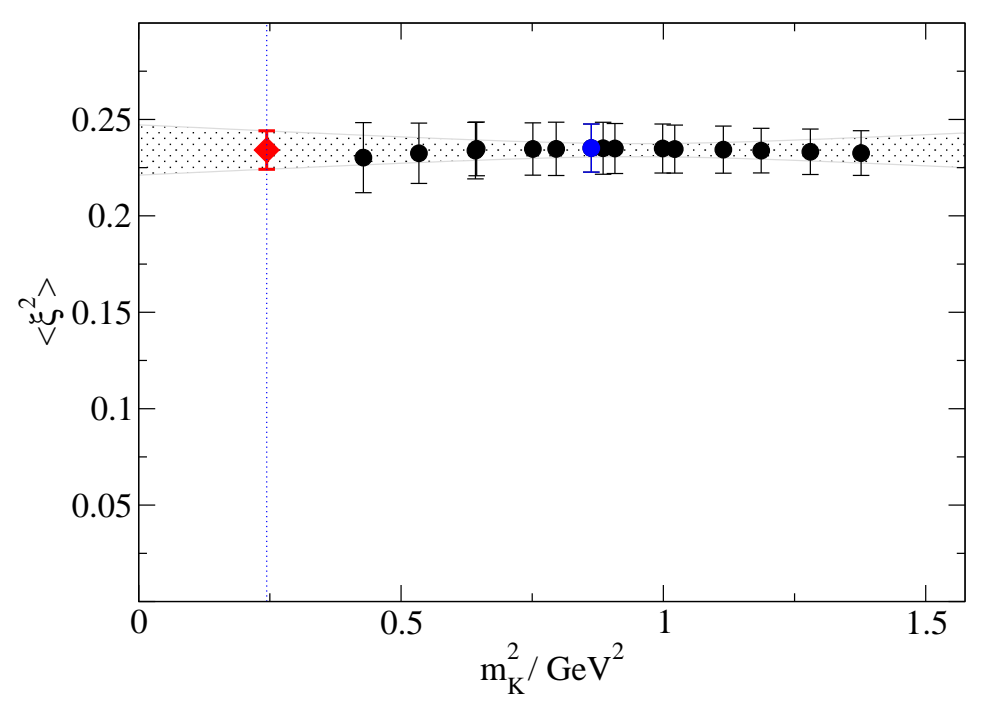

Figure 3: Extrapolation of the second moment of the kaon distribution amplitude, $\left\langle\xi^{2}\right\rangle_{K}$ at $\mu^{2}=4 \mathrm{GeV}^{2}$, at $\beta=5.29, \kappa_{\text {sea }}=\kappa_{\text {light }}=0.1359$ and several values of $\kappa_{\text {val }}=\kappa_{\text {heavy }}$. The extrapolation to the physical value is shown at the vertical line. The blue data point indicates the degenerate mass case, $\kappa_{\text {heavy }}=\kappa_{\text {light }}$.

\section{Summary}

We have performed a calculation of the first two moments of the distribution amplitudes of pseudoscalar mesons. In the case of the pion we have found agreement with previous sum rule calculations and phenomenological evaluations from experimental data. In the case of the kaon we have also found agreement with previous sum rule estimates, but with smaller statistical errors. The uncertainty due to the continuum extrapolation has been estimated and found to be small.

\section{Acknowledgments}

The numerical calculations have been done on the Hitachi SR8000 at LRZ (Munich), on the Cray T3E at EPCC (Edinburgh) under PPARC grant PPA/G/S/1998/00777 [15] and on the APEmille and APEnext at NIC/DESY (Zeuthen). This work was supported in part by the DFG under contract FOR 465 (Forschergruppe Gitter-Hadronen-Phänomenologie) and in part by the EU Integrated Infrastructure Initiative Hadron Physics (I3HP) under contract number RII3-CT-2004506078. W.S. thanks the Physics Department of the National Taiwan University for their hospitality and Jiunn-Wei Chen for valuable remarks and discussions.

\section{References}

[1] S. J. Brodsky and G. P. Lepage, Adv. Ser. Direct. High Energy Phys. 5 (1989) 93.

[2] V. M. Braun et al., arXiv:hep-lat/0606012. 


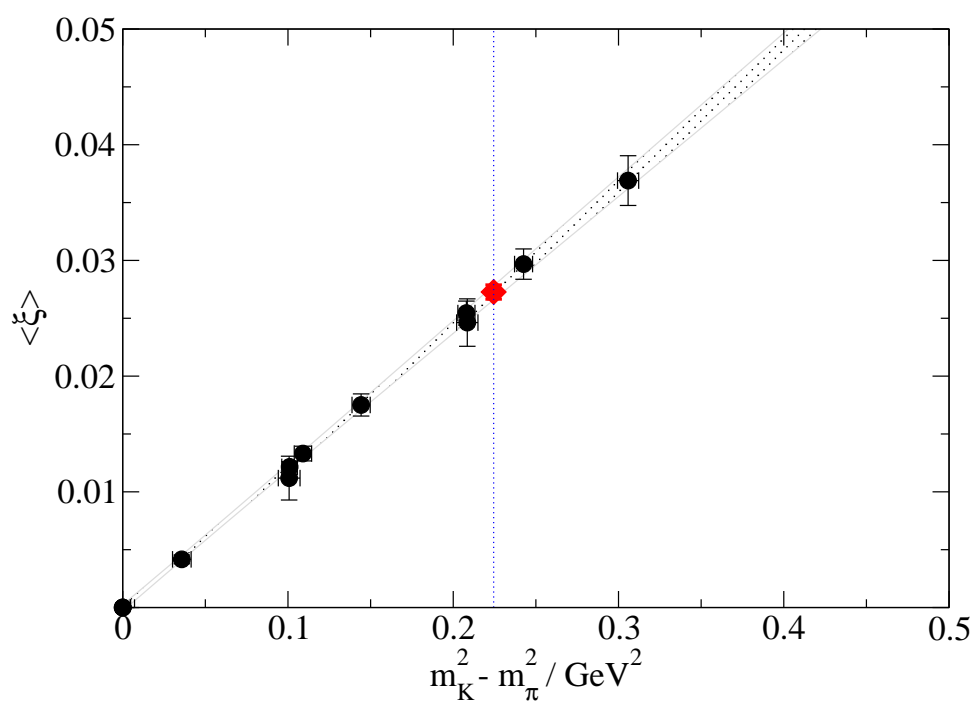

Figure 4: Interpolation of the first moment of the kaon DA, $\langle\xi\rangle_{K}$ at $\beta=5.29, \kappa_{\text {sea }}=0.1359$ as obtained from $\mathscr{O}_{44}^{b}$. The physical point corresponding is indicated by the vertical line, the value obtained by a linear interpolation.

[3] P. A. Boyle, M. A. Donnellan, J. M. Flynn, A. Jüttner, J. Noaki, C. T. Sachrajda and R. J. Tweedie [UKQCD Collaboration], arXiv:hep-lat/0607018; A. Jüttner et al., these proceedings, arXiv:hep-lat/0610025; A. Jüttner et al., in preparation.

[4] M. Göckeler, R. Horsley, A. C. Irving, D. Pleiter, P. E. L. Rakow, G. Schierholz and H. Stüben, Phys. Rev. D 73, 014513 (2006).

[5] M. Göckeler, R. Horsley, H. Perlt, P. E. L. Rakow, A. Schäfer, G. Schierholz and A. Schiller, arXiv:hep-lat/0605002.

[6] M. Göckeler, R. Horsley, D. Pleiter, P. E. L. Rakow and G. Schierholz [QCDSF Collaboration], Phys. Rev. D 71, 114511 (2005).

[7] J. W. Chen and I. W. Stewart, Phys. Rev. Lett. 92, 202001 (2004).

[8] R. G. Edwards et al. [LHPC Collaboration], Phys. Rev. Lett. 96 (2006) 052001; A. Ali Khan et al., arXiv:hep-lat/0603028.

[9] L. Del Debbio, M. Di Pierro and A. Dougall, Nucl. Phys. Proc. Suppl. 119, 416 (2003).

[10] M. Diehl, P. Kroll and C. Vogt, Eur. Phys. J. C 22, 439 (2001).

[11] V. L. Chernyak and A. R. Zhitnitsky, Phys. Rept. 112 (1984) 173.

[12] P. Ball, V. M. Braun and A. Lenz, JHEP 0605, 004 (2006).

[13] A. P. Bakulev, S. V. Mikhailov and N. G. Stefanis, Phys. Lett. B 578, 91 (2004); A. P. Bakulev, S. V. Mikhailov and N. G. Stefanis, Phys. Rev. D 67, 074012 (2003).

[14] A. Khodjamirian, T. Mannel and M. Melcher, Phys. Rev. D 70, 094002 (2004).

[15] C. R. Allton et al. [UKQCD Collaboration], Phys. Rev. D 65, 054502 (2002). 